\title{
Measurement of Relative Metastable Level Population of Gd Atoms in Hollow Cathode Lamp with LIF Method
}

\author{
Seyed Hassan Nabavi, Ata Koohian \\ Faculty of Physics, Tehran University, Tehran, Iran \\ E-mail:koohian@ut.ac.ir \\ Received February 5, 2010; revised March 29, 2010; accepted March 31, 2010
}

\begin{abstract}
Relative metastable level population of metal plasma having low-lying metastable states departs from equilibrium value. It needs to be experimentally investigated. This paper reports the use of hollow cathode lamp based Laser Induced Fluorescence (LIF) spectroscopy technique to measure Relative metastable level population of metal in a plasma produced by a hollow cathode lamp. The relative population of ground state and $533 \mathrm{~cm}^{-1}$ levels of Gd atoms in hollow cathode lamp is measured with LIF method.
\end{abstract}

Keywords: Gadolinium, Fluorescence, Population

\section{Introduction}

Gd metal has widespread applications in medical, astronomy and nuclear industries. Work on various parameters of this metal has drawn the attention of many researchers $[1,2]$. Hollow cathode lamps are mainly used for investigating various parameters of Gd Metals [3]. The scattered atoms of the Gd metal in the lamp have metastable levels, with low energy and high life time. These levels will be populated due to number of collisions such as the collisions of atoms with each other, collision of atoms with electrons, and collision of atoms with lamp's wall. The population's measurements at these levels are of a significant importance, especially in those experiments related to laser and material interactions where atoms from metastable levels are excited to other levels which have higher energies. Therefore the knowledge of knowing when the states are fully populated is essential in analyzing these types of activities. There are different methods for the measurement of level's populations, like absorption spectrum measurement which is the most current method. However one of the main problems with this method is its high optical noise [4]. In this paper, the use of inductive fluorescence method is proposed for the calculation of the relative population of the Gd metastable levels.

\section{Laser Induced fluorescence (LIF) in lamp}

The Gd metal in a hallow cathode lamp is scattered from the cathode by buffer gas atoms and is vaporized in the lamp. The energetic electrons, ions, excited neutral atoms of the buffer gas collide with Gd vapor and cause the population and depopulation Gd levels. These processes create a new distribution of levels population in $\mathrm{Gd}$ atoms [5]. If there were no electrons in the lamp, Boltzmann distribution could be used for the estimation of the population distribution. However the existence of electrons in the lamp makes the Boltzmann distribution to be a void distribution for the population. This suggests that a different method should be used for obtaining the distribution.

The LIF method is one of the best methods for obtaining the population distribution in such cases with low atomic density and high disorderly. LIF is a process where atoms are excited to higher electronic energy states via laser absorption and induces fluorescence radiation.

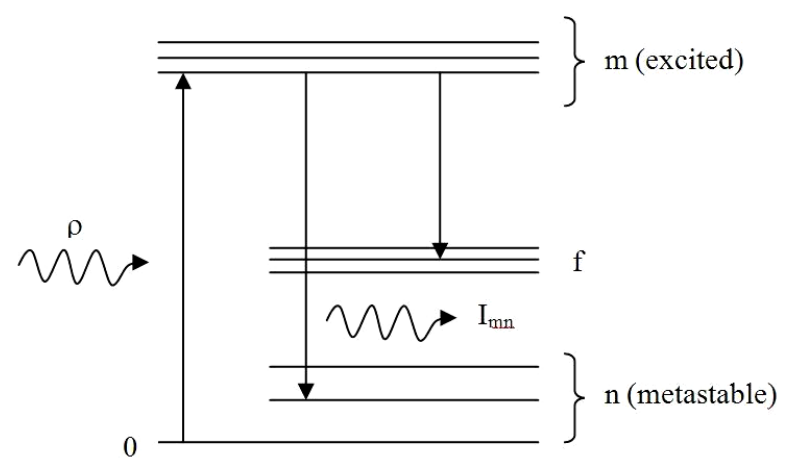

Figure 1. Some possible transitions in LIF. 
The intensity of this fluorescence is dependent on the absorption density. Typically fluorescence occurs at wavelengths grater than or equal to laser wavelength. Metastable levels (Figure 1) can be excited to higher levels by a visible laser lines and then the fluorescence (Figure 1) can be detected by a monochromator. Fluorescence intensity is [6]:

$$
I_{m n}=A_{m n} N_{m} h v_{m n} \eta
$$

where $N_{m}$ is the Upper level population, $A_{m n}$ is transition probability from $\mathrm{m}$ to $\mathrm{n}, h v_{m n}$ is the energy of this transition and $\eta$ is the correction coefficient related to detection systems like photon multiplier (PMT) and the grating in monochromator.

$$
\eta=\eta_{P M T} \times \eta_{\text {grating }}
$$

where $\eta_{P M . T}$ and $\eta_{\text {grating }}$ are the correction coefficients. $N_{n}$ can be obtained by rate equations,

$$
\begin{gathered}
\frac{d N_{m}}{d t}=-N_{m} R_{m}-N_{m} B_{n m} \rho \\
\frac{d N_{n}}{d t}=N_{m} B_{n m} \rho-N_{m} B_{n m} \rho+N_{m} A_{m n}-\frac{N_{n}}{\tau_{n}}
\end{gathered}
$$

where, $B$ is the Einstein coefficient of the transitions, $\rho$ is the density of the photons that react with atoms, $\tau_{n}$ is the life time of the low level, and $R_{m}$ is the rate of radiation and non radiation fall downs from upper level $\mathrm{m}$ (Figure 1).

$$
R_{m}=A_{m n}+A_{m} \rightarrow f \neq n+R_{\text {collision }}
$$

By considering the fact that the lower level $n$, is metastable level, so $1 / / \tau_{n}$ goes to zero and the relative population of levels will be calculated from the numerical solutions of (3).

\section{Experimental Method}

Figure 2 shows the experimental arrangement for the measuring the relative population. a Ring dye laser beam which is capable of scanning $30 \mathrm{GHz}$ around a wavelength is focused into hollow cathode lamp with Gd metals as its cathode. The buffer gas inside this lamp is $\mathrm{Ne}$ with the pressure 3 torr and the maximum current that can pass through this lamp is $15 \mathrm{~mA}$. With the gas pressure of 3torr and $10 \mathrm{~mA}$ electrical current the $R_{\text {collision }}{ }^{-1}$ in (4) is about 50 ns [6].

By sweeping a range of wavelengths and setting the system wavelength to a desirable value, the $\mathrm{Gd}$ atoms are excited to upper levels and then transit to lower levels (Figure 1). Fluorescence induced by these transitions are focused into entrance window of monochromotor by a short focal length lens. The focused beam will exit the monochromotor after hitting the holographic grating
(1200 1/mil). The angel of grating relative to the entrance light must be arranged in a way that the desired Florescence line is selected. This beam will enter PMT and will be amplified electrically. This will allow the observation of the beam on oscilloscope. The measurements of the relative population of the ground state and the $533 \mathrm{~cm}^{-1}$ level are required. These levels have wavelengths of $5618 \mathrm{~A}^{\circ}$ and $5791 \mathrm{~A}^{\circ}$ respectively and will be excited to $17795 \mathrm{~cm}^{-1}$ level and then the radiation from the atomic transition to level $215 \mathrm{~cm}^{-1}$ can be observed on the PMT (Figure 3).

By observing the number of fluorescence photon at the excited wavelengths of levels 0 and $533 \mathrm{Cm}^{-1}$ and by putting these numbers into (3) and solving these equations simultaneously in the steady state conditions, the relative population of the two levels is obtained.

\section{Conclusions}

HCL based LIF has been implemented to measure relative level population of Gadolinium. the ground and $533 \mathrm{Cm}^{-1}$ metastable states. in a HCL. Utilizing the emission of HCL provides for relative atom density measurement. This becomes particularly useful when there is a low fluorescence signal in the atomic data. It is a good technique using the LIF emission from a hollow cathode that can be utilized to measure the relative atom density. By using the experimental results and standard tables [7] a value around 2 , i.e. $\mathrm{N}_{533} / \mathrm{N}_{0}=2 \pm 15 \%$ for relative population of the levels $533 \mathrm{Cm}^{-1}$ and $0 \mathrm{Cm}^{-1}$, was obtained, which means due to existence of electrons and their collisions with $\mathrm{Gd}$ atoms the population distribution pattern of level's does not follow the Boltzmann law any more.

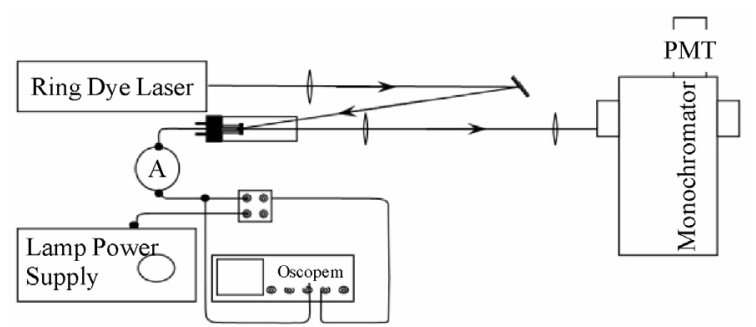

Figure 2. Experimental arrangement for measuring LIF signal.
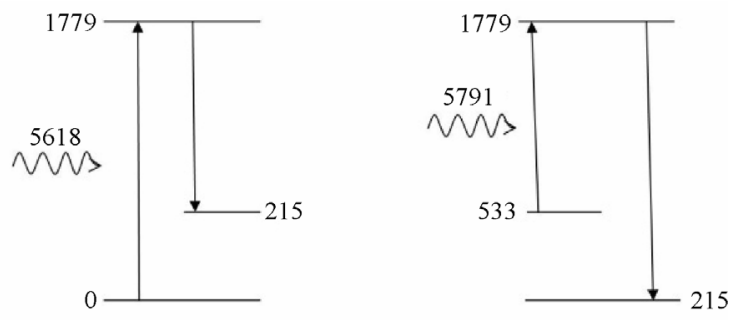

Figure 3. Engaged levels in experiment. 
The LIF method with its simple experimental arrangement has a significantly high signal to noise ratio compared to similar methods like optogalvanic or absorption methods. In this experiment the measurements have been conducted using Gd hollow cathode lamp for the first time and it is suggested that this measurements can be obtained for other states by using lasers with different wavelength.

\section{References}

[1] E. Bi'emont, G. Kohnen and P. Quinet, "Transition Probabilities in Gd III," Astronomy and Astrophysics, Vol. 393, No. 2, 2002, pp. 717-720.

[2] S. A. Enger, A. Rezaei and P. Munck, "Godolinium Neutron Capture Brachythrapy (GDNCB), A New Treatment Method for Intravascular Brachythrapy," Medical Physics, Vol. 33, No. 1, 2006, pp. 46-51.
[3] A. Majumder, B. Dikshit, M. S. Bhatia and V. K. Mago, "Use of Multiwavelength Emission from Hollow Cathode Lamp for Measurement of State Resolved Atom Density of Metal Vapor Produced by Electron Beam Evaporation," Review of Science Instrument, Vol. 79, No. 9, 2008, pp. 1-7.

[4] A. Okamoto, T. Kobuchi, S. Kitajima and M. Sasao, "Study of Metastable Population Density in a Hollow Cathode Helium Discharge," Plasma and Fusion Research, Vol. 2, 2007, p. 29.

[5] R. Payling, D. G. Gones and A. Bengtson, "Glow Discharge Optical Emission Spectroscopy," John Wiley, 1997, pp. 343-347.

[6] W. Demtroder, "Laser Spectroscopy," Springer, 1996.

[7] C. H. Corliss and W. A. Bozman, "Experimental Transition Probabilities for Spectral Line of Seventy Elements," 1962 